\title{
Temperature Sensitivity of Polymer Fiber Microlasers
}

\author{
Chun WANG ${ }^{1}$, Xiaojuan ZHANG ${ }^{2}$, Jiajun $\mathrm{MA}^{3}$, Kang XIE${ }^{4}$, \\ Junxi ZHANG ${ }^{1}$, and Zhijia $\mathrm{HU}^{2,3^{*}}$ \\ ${ }^{1}$ Laboratory of Optical Fibers and Micro-nano Photonics, School of Instrument Science and Opto-electronics \\ Engineering, Hefei University of Technology, Hefei 230009, China \\ ${ }^{2}$ Information Materials and Intelligent Sensing Laboratory of Anhui Province, Key Laboratory of Opto-Electronic \\ Information Acquisition and Manipulation of Ministry of Education, School of Physics and Optoelectronics Engineering, \\ Anhui University, Hefei 230601, China \\ ${ }^{3}$ State Key Laboratory of Environmental Friendly Energy Materials, Southwest University of Science and Technology, \\ Mianyang 621000, China \\ ${ }^{4}$ School of Opto-Electronic Engineering, Zaozhuang University, Zaozhuang 277160, China \\ "Corresponding author: Zhijia HUＥ-mail: zhijiahu@hfut.edu.cn
}

\begin{abstract}
There are many kinds of materials or methods used to make optical microcavities, and they have many different geometric structures. And electrospinning technique has become a very convenient and easy one to prepare polymer fiber. Based on this situation, PM597-doped polymer solution was prepared into high-performance fibers with different diameters by electrospinning technology in our work. In order to better study the temperature sensing of polymer fiber whispering gallery mode, we have placed it on two different substrates with gold and aluminum. A $532 \mathrm{~nm}$ pulsed laser beam was used to excite a single fiber in the radial direction, then the whispering gallery mode (WGM) laser was observed and the distribution of WGM was determined by theoretical calculations. The threshold of samples on aluminum substrate is $0.4 \mu \mathrm{J}$. In addition, it is found that the samples on aluminum substrate performed better in temperature sensing, and the value is $0.13 \mathrm{~nm} /{ }^{\circ} \mathrm{C}$. As a result, WGM polymer fiber microcavities on aluminum substrate made by electrospinning technology have very broad development prospects in biosensing, optical pump lasers and other applications.
\end{abstract}

Keywords: Whispering gallery mode; gold substrate; electrospinning

Citation: Chun WANG, Xiaojuan ZHANG, Jiajun MA, Kang XIE, Junxi ZHANG, and Zhijia HU, "Temperature Sensitivity of Polymer Fiber Microlasers," Photonic Sensors, 2022, 12(3): 220307.

\section{Introduction}

The whispering gallery mode (WGM) refers to a phenomenon when the light beam propagates along the geometric inner wall and continuous total reflection occurring in a geometric structure with rotation symmetry. If the optical path of the light beam that walks around the boundary of the geometric structure satisfies an integer multiple of the wavelength, an interference-enhancing phenomenon or resonance phenomenon will occur. The ring structure used to constrain the light field is called the whispering gallery mode optical microcavity. Tunable optical micro-resonators with

Received: 29 July 2021 / Revised: 24 September 2021

(C) The Author(s)2022. This article is published with open access at Springerlink.com

DOI: 10.1007/s13320-021-0647-0

Article type: Regular 
high optical quality, small size, and flexibility are the key enabling elements for many photonic integrated circuits. WGM optical microcavity has always been the focus of research because of its higher quality factor, smaller mode volume, and significantly enhanced interaction between light matters. Nowadays, WGM optical microcavities are made of various materials, such as silica, silicon, silicon nitride, and polymer [1]. Furthermore, the common geometric structures are microspheres, microbubbles, microdisks, microtubes, microrings, and optical fibers [2]. Since polymer optical fiber is low cost, mechanical flexibility, and easy fabrication, it has become a good candidate medium for a low-threshold lasing.

There are various of ways to make polymer fibers as media to boost WGM lasing. Linslal et al. [3] reported the WGM laser emission from a Rhodamine $\mathrm{B}(\mathrm{RhB})$ dye doped graded index (GI) polymer fiber fabricated by interfacial gel polymerization technique. Ta et al. [4] used a metal rod with a sharp tip immersing into the droplet and moved to make microfibers doped with Rhodamine 6G (R6G) and epoxy resin, and they have analyzed the WGM lasing mechanism. Anand et al. [5] carried out lasing studies on dye-doped hollow fibers with different radii with optical pumping. Linslal et al. [6] used polymer optical fiber drawing tower to fabricate RhB-doped polymer fibers and observed the wavelength tuning of WGM. Among these methods, the electrospinning technology has gained wide-spread interest in manufacture of polymer fibers because of its low cost and good control capability [5].

Low threshold is one of the most significant features of WGM lasers, and numerous studies have shown that polymer fiber microcavities have a distinct low threshold than other geometric structures Anand et al. [5] have studied WGM laser emission from a RhB-doped poly (methyl methacrylate) (PMMA) hollow optical fiber and the threshold was $0.25 \mathrm{~mJ}$. Chen et al. [7] achieved bidirectional tuning of WGM lasing wavelength by encapsulating the dye-doped microfibers with polydimethylsiloxane (PDMS) elastomer and the threshold was $0.97 \mu \mathrm{J}$. Ta et al. [4] made microfibers doped with Rhodamine 6G (R6G) and epoxy resin and they have analyzed the threshold of WGM laser was $0.4 \mu \mathrm{J}$. Thus polymer fiber fabricated by electrospinning is deserved to research.

Tuning of the WGM microcavities is important in many applications, such as cavity quantum electrodynamics, biological sensing, parity-time (PT) symmetry, and electromagnetically inducing transparency [1]. There are many studies in other geometric structures. Martín et al. [8] prepared microspheres of $\mathrm{Nd}^{3+}$ doped barium titano silicate glass, and temperature sensing and WGM were observed. Li et al. [9] obtained high temperature WGM lasing sensors with $0.151 \mathrm{~nm} / \mathrm{K}$ through coating a layer of PDMS on the surface of a silica toroidal microresonator on a silicon wafer. Zhang et al. [10] fabricated a fiber-coupled WGM microsphere resonator with temperature sensitivity of $6.82 \mathrm{pm} /{ }^{\circ} \mathrm{C}$ in the process of heating and $7.38 \mathrm{pm} /{ }^{\circ} \mathrm{C}$ in the process of cooling. To the best of our knowledge, there are not much more researches on temperature sensitivities of WGM fiber microcavities. Anand et al. [5] have studied temperature-induced tuning of WGM laser emission from a RhB-doped poly (methyl methacrylate) (PMMA) hollow optical fiber with a sensitivity of $0.011 \mathrm{~nm} /{ }^{\circ} \mathrm{C}$. Thus, WGM polymer fiber microlasers have a great potential application in temperature sensing.

In this paper, we fabricated polymer fiber microcavities with the diameters ranging from $4 \mu \mathrm{m}$ to $40 \mu \mathrm{m}$ by electrospinning. The surface plasmons (SPs) of metal substrate coupling with WGM polymer fiber microcavities are a possibility for temperature sensing improvement. Given this, all these microcavities were placed on gold substrate and aluminum substrate, respectively. We researched the effect of metal substrate on the WGM lasing emission and sensitivity. Besides, it has been 
observed lasing emission for the two samples under SPs, both of which have low thresholds due to SPs of metal substrates with the values of $0.2 \mu \mathrm{J}$ and $0.4 \mu \mathrm{J}$. Moreover, it has been investigated in temperature sensing characteristics of fiber on the two substrates. A wavelength tuning range of fiber on the aluminum substrate is $0.8 \mathrm{~nm}$ varying the temperature from $27{ }^{\circ} \mathrm{C}$ to $33{ }^{\circ} \mathrm{C}$, and the high temperature sensitivity is $0.13 \mathrm{~nm} /{ }^{\circ} \mathrm{C}$. The temperature sensitivity of fiber on the gold substrate is $0.07 \mathrm{~nm} /{ }^{\circ} \mathrm{C}$ at the same temperature variation range.

\section{Materials and fabrications}

In this experiment, we chose the electrospinning technology to fabricate polymer fibers on different substrates. Since PMMA possesses a higher thermooptic coefficient and thermo-expansion coefficient, the solution used to make samples was mixed with

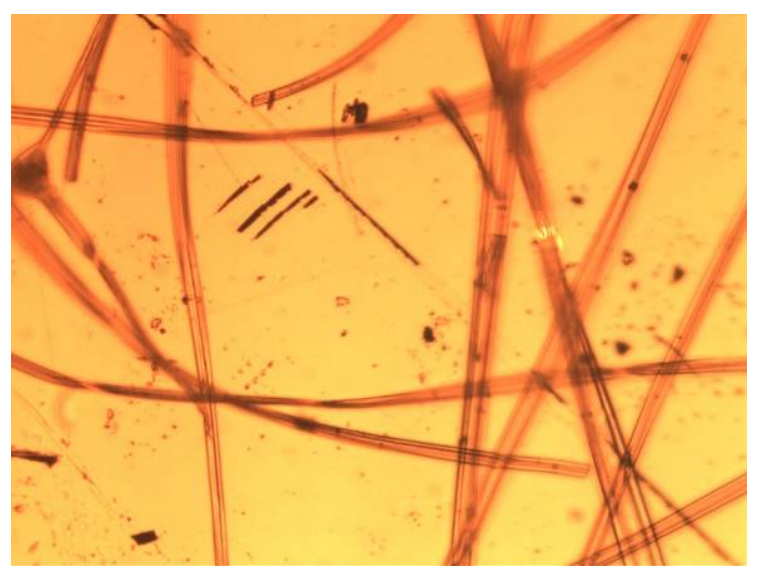

(a)

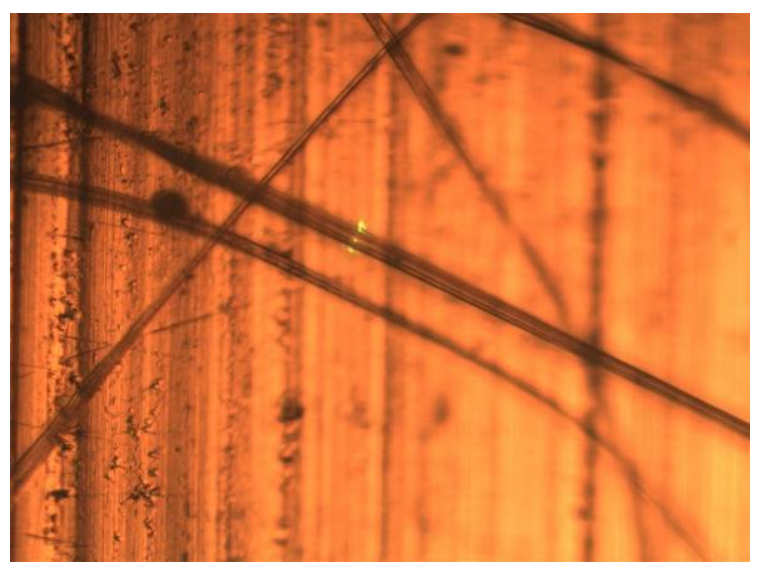

(c)
38 wt.\% PMMA and 0.4 wt.\% Pyrromethene 597 (PM597) in $10 \mathrm{~mL}$ acetone. A $5 \mathrm{~mL}$ syringe, which was attached to a $0.9 \mathrm{~mm}$ needle, was used to load the solution. A metal collector was placed directly opposite with the distance of $20 \mathrm{~cm}$. The high voltages between them were in the range of $-30 \mathrm{kV}$ to $50 \mathrm{kV}$, and the positive pole was connected to the tip of the needle while the other pole was connected to the metal collector. The feed rate of solutions was controlled at $0.08 \mathrm{~mm} / \mathrm{min}$ through the above syringe. Polymer fibers were fabricated on gold substrate and aluminum substrate.

The diameters of polymer fibers ranges from $4 \mu \mathrm{m}$ to $40 \mu \mathrm{m}$, and the majority of diameter is between $15 \mu \mathrm{m}$ and $25 \mu \mathrm{m}$. Figure 1 illustrates the images of single polymer fiber pumped by $532 \mathrm{~nm}$ laser with different diameters on gold and aluminum substrates.

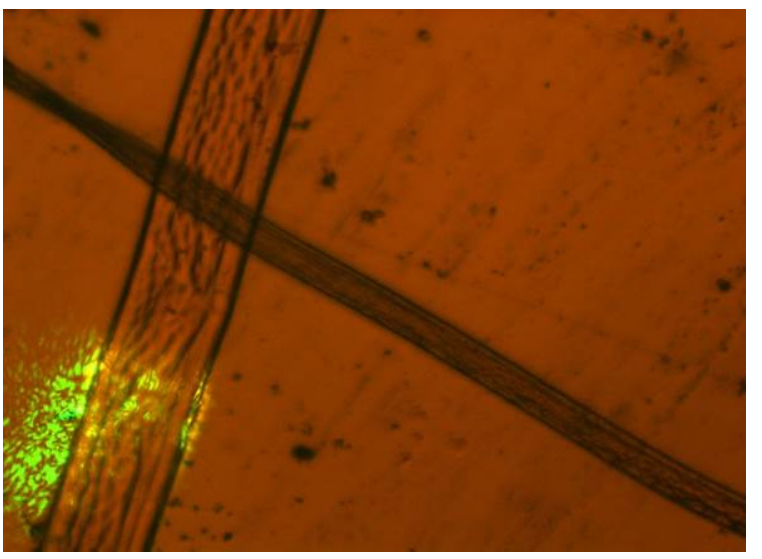

(b)

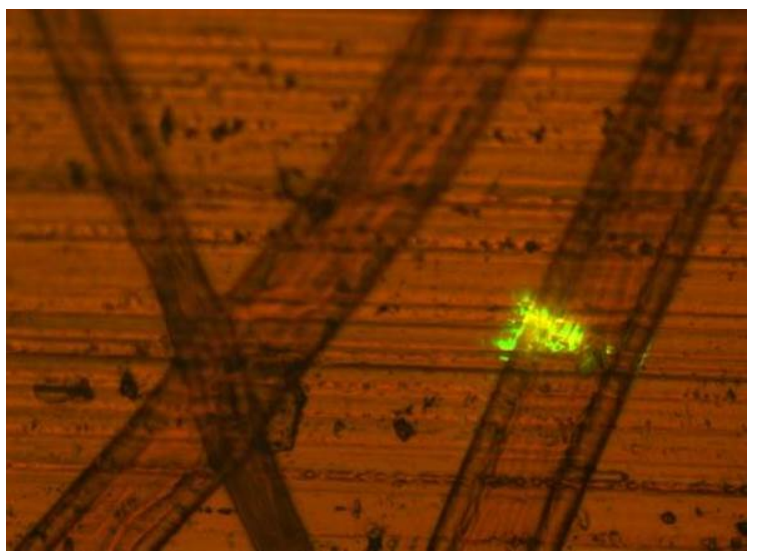

(d)

Fig. 1 Images of (a) $10 \mu \mathrm{m}$ and (b) $30 \mu \mathrm{m}$ fiber on gold substrate; (c) $10 \mu \mathrm{m}$ and (d) $40 \mu \mathrm{m}$ fiber on aluminum substrate. 


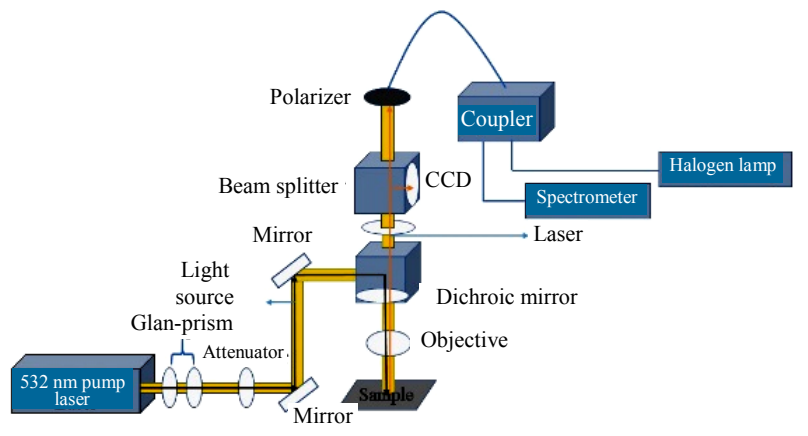

Fig. 2 Microscope lasing measurement setup.

Figure 2 shows the experimental setup to measure WGM lasing. The pumping source is a Q-switched Nd: YAG laser, and it can output a round spot with wavelength of $532 \mathrm{~nm}$ (pulse duration of $10 \mathrm{~ns}$, repetition rate of $10 \mathrm{~Hz}$, and spot diameter of $30 \mu \mathrm{m}$ ). The pump pulse energy and polarization were controlled by a Glan prism group. The polarizer near the pumping source was used to change pump energy, and another one determines the polarized states of the pump beam. An attenuator for weakening the pump pulse energy was placed to the back. Then two mirrors placed at an angle of $45^{\circ}$ were used to introduce the pumping laser path into the micro spectrum system. Through a $532 \mathrm{~nm}$ total reflection dichroic mirror and a focused objective, the pumping laser was focused onto the samples. Then the emission light emitted from single fiber entered the micro spectrum system, and was collected by the microscope objective and guided to a beam splitter where $50 \%$ of the lasing emission was sent to the charge coupled device (CCD) camera to obtain the image of the sample. The remaining $50 \%$ of the laser emission passed through the polarizer and was received by the connected common fiber, and then the polarization of the WGM lasing emission could be detected by rotating the polarizer (the angle between the polarizer's axis and $X$-axis was defined as $\theta$ ). The fiber spectrometer (QE65PRO, Ocean Optics, resolution $\sim 0.4 \mathrm{~nm}$, integration time $100 \mathrm{~ms}$ ) was used to collect spectra and the halogen lamp was used to highlight the pump position which was connected to a common fiber through a $1 \times 2$ multimode fiber coupler.

\section{Results and discussion}

Figure 3 describes WGM characteristics of the single polymer fiber with diameter of $18 \mu \mathrm{m}$ on gold substrate and $20 \mu \mathrm{m}$ on aluminum substrate. As shown in Figs. 3(a) and 3(b), the lasing intensity increases with an increase in pump energy for polymer fibers on the gold and aluminum substrates. In our experiment, when the pump energy is $0.5 \mu \mathrm{J}$, the main lasing peak appears at $575.8 \mathrm{~nm}$ with the full width half maximum (FWHM) of $\sim 0.3 \mathrm{~nm}$ for fiber on the gold substrate. While the main lasing peak appears at $579.2 \mathrm{~nm}$ with the FWHM of $\sim 0.4 \mathrm{~nm}$ for fiber on the aluminum substrate. As shown in Figs. 3(c) and 3(d), the thresholds are about $0.2 \mu \mathrm{J}$ and $0.4 \mu \mathrm{J}$, respectively. It can be clearly observed that the difference of substrates has an impact on the threshold of WGM lasing. Supplementary studies have been conducted on WGM lasing for polymer fibers with different diameters. It is found that the thresholds for different diameter cases are basically constant. In previous reports, it offered a relatively suitable explanation. $\mathrm{Xu}$ et al. [11] achieved an improvement in WGM lasing of $\mathrm{ZnO}$ microtubes through SPs coupling of $\mathrm{Au}$, and found that the threshold value was reduced. Under light excitation, the SPs of metal substrate got excited, which increased the evanescent field of WGM of polymer fiber microresonator $[11,12]$. And the SPs are sensitive to the materials and the permittivities of the surrounding environment, since the dielectric constants of $\mathrm{Au}$ is bigger than $\mathrm{Al}$ in visible light range and it contributes to stronger SPs [11-13], the threshold of fiber on gold substrate is lower than that of aluminum. According to [4], [5], and [7], the thresholds in our work are $0.2 \mu \mathrm{J}$ for gold and $0.4 \mu \mathrm{J}$ for aluminum substrate, which are both smaller or equal to the data presented in references. 


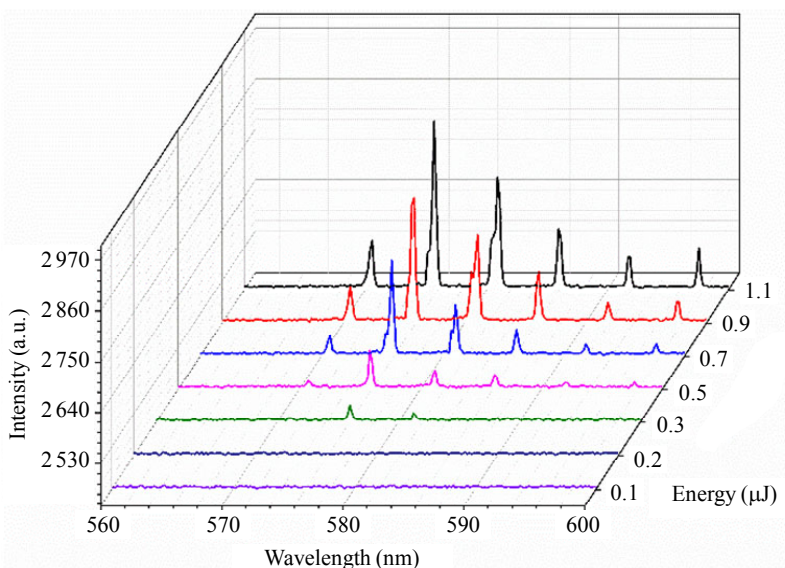

(a)

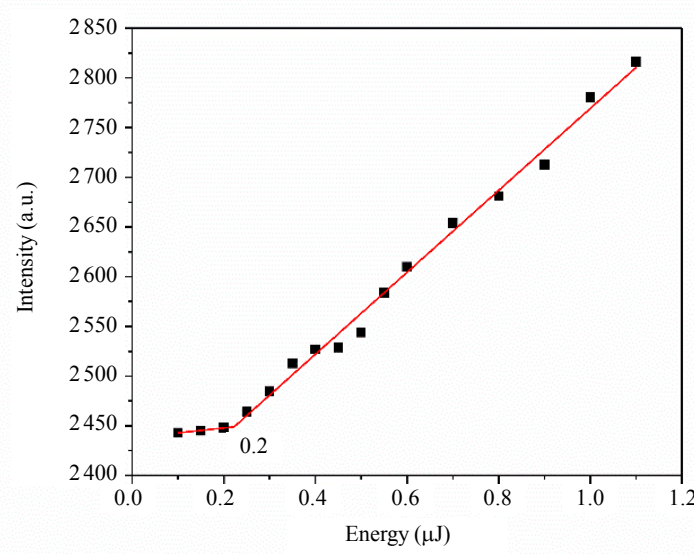

(c)

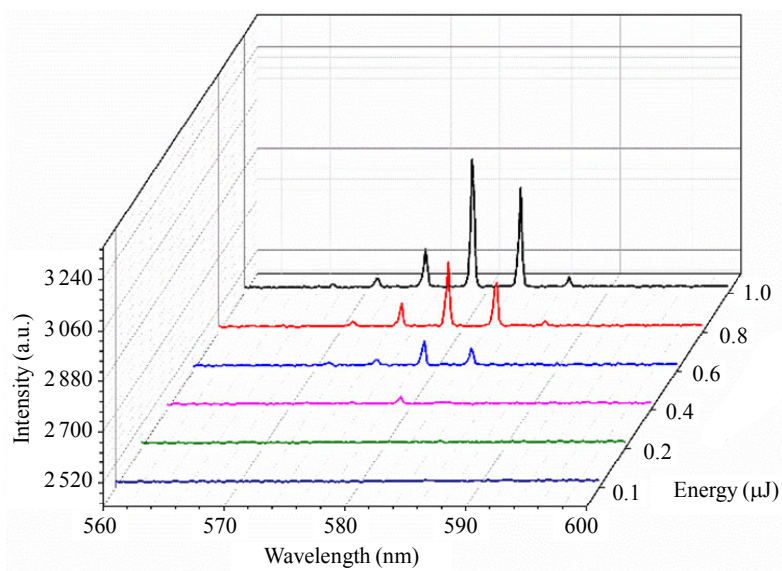

(b)

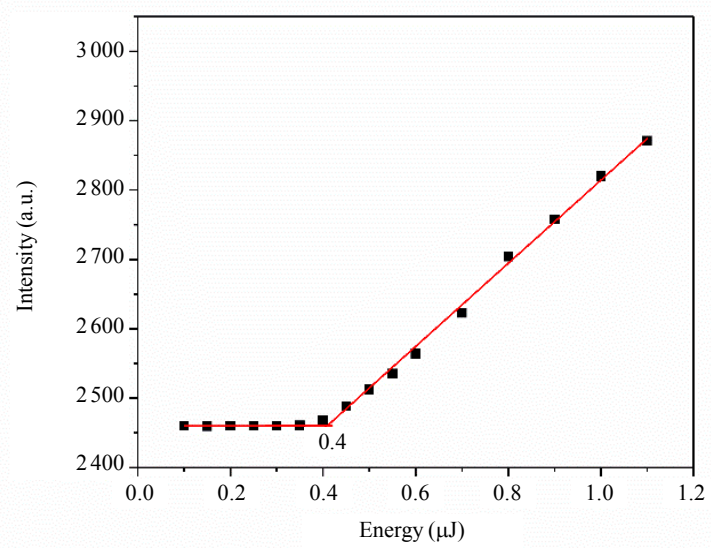

(d)

Fig. 3 WGM lasing emission spectra of single polymer fiber on (a) gold substrate and (b) aluminum substrate with different energy. WGM lasing emission intensity as a function of pumping energy on (c) gold substrate and (d) aluminum substrate.

Additionally, the polarization characteristics of the WGM lasing emission is another factor which is deserved to study. There are two polarization modes that are transverse magnetic (TM) and transverse electric (TE) modes based on the polarization direction of the pump beams in the fiber [4-7]. When the electric field is parallel to the length axis of the fiber, the lasing emission is a transverse magnetic TM wave that forms a special axially polarization emission, and vice versa the radial emission formed perpendicularly is TE wave $[4,7,14]$. And we used the polarization measurement device in Fig. 2 to characterize the polarization of the WGM laser. The initial angle expressed as the direction of polarizer was parallel to $X$-axis, thus $\theta$ was 0 . The diameter of the polymer fiber was $\sim 11 \mu \mathrm{m}$ both on gold and aluminum substrates. Figures 4(a) and 4(c) show the lasing intensity as a function of rotation angle of the polarizer. The lasing emission intensity reaches its maximum in the condition that the polarizer is set along $X$-axis $\left(\theta=0^{\circ}\right.$ and $\left.180^{\circ}\right)$. The conclusion that the lasing emission is only TM mode is based on the fact that the intensity variation is very consistent with Malus's law $\left[I=I_{0}\left(\cos ^{2} \theta\right)\right]$ both on the two samples. In order to determine the laser mechanism, we further analyzed the laser characteristics. The lasing modes $(\mathrm{m})$ can be calculated from the following formula $[7,14-17]$ : 


$$
\lambda^{-1}\left(R, n_{1}, n_{r}, r, m\right)=\frac{1}{2 \pi R n_{1}}\left[\begin{array}{l}
m+\frac{1}{2}+2^{-\frac{1}{3}} a(r)\left(m+\frac{1}{2}\right)^{\frac{1}{3}}-\frac{L}{\left(n_{r}^{2}-1\right)^{\frac{1}{2}}}+ \\
\frac{3}{10} 2^{-\frac{2}{3}} a^{2}(r)\left(m+\frac{1}{2}\right)^{-\frac{1}{3}}-2^{-\frac{1}{3}} L\left(n_{r}^{2}-\frac{2}{3} L^{2}\right) \frac{a(r)\left(m+\frac{1}{2}\right)^{-\frac{2}{3}}}{\left(n_{r}^{2}-1\right)^{\frac{3}{2}}}
\end{array}\right]
$$

where $\lambda$ is the resonant wavelength, $R$ is the radius of the single fiber microcavity, $n_{1}$ is the refractive index of fiber, $n_{2}$ is the refractive index of surrounding medium, and $n_{r}=n_{1} / n_{2} . L$ is the polarization characteristic coefficient, $L=n_{r} \quad$ is for TE modes, and $L=n_{r}^{-1}$ is for TM modes. $a(r)$ is the root of the Airy function, where $r$ is the radial mode number, and $m$ is the mode number. Figure 4 depicts the WGM lasing polarization characteristics for PM597-doped single fiber at pump energy of $0.9 \mu \mathrm{J}$. The refractive index of single fiber and

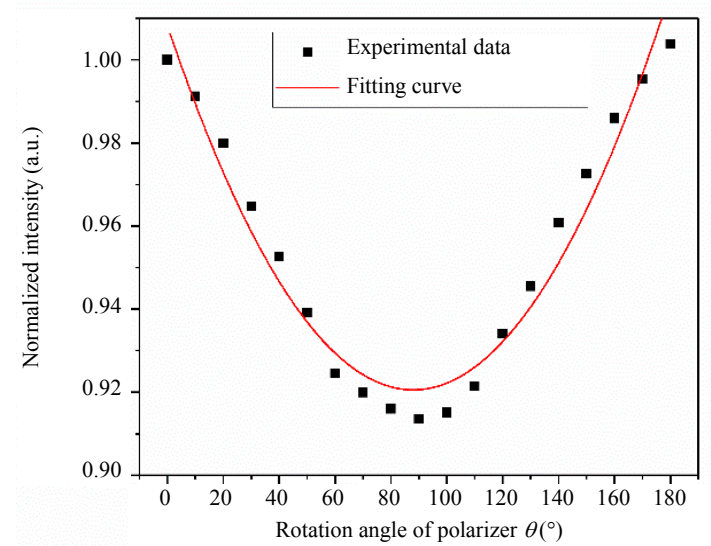

(a)

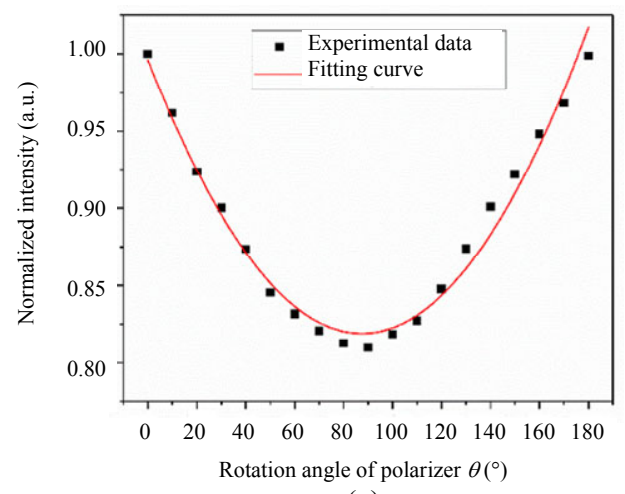

(c) surrounding air are $1.487\left(n_{1}\right)$ and $1\left(n_{2}\right)$ in our experiment. The WGM peaks belongs to the first-order $(r=1)$ TM mode theoretically, and the corresponding mode numbers $m$ are found to be 92-96 and 99-101 for polymer fibers on gold and aluminum substrates, respectively. These values closely match the laser peaks in the corresponding spectra shown in Figs.4(b) and 4(d), thus validating the WGM lasing emission mechanism. From Fig. 4, it can be shown that the substrate has no effect on the lasing mode, while it mainly plays an effect on the mode numbers.

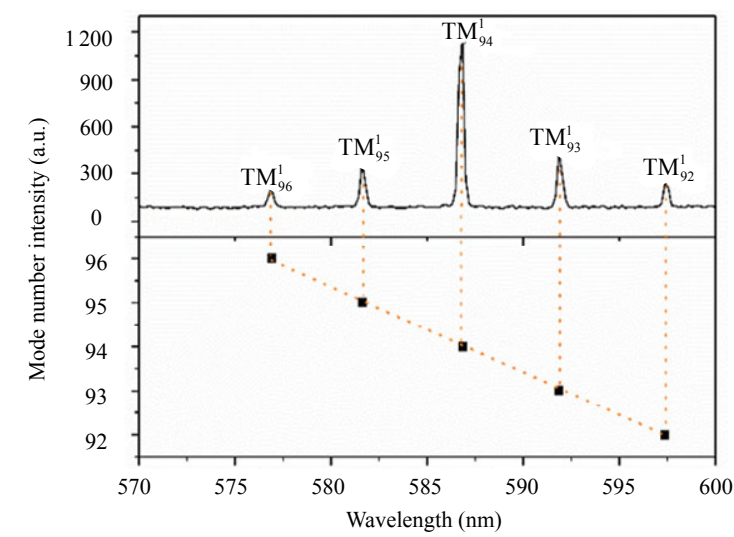

(b)

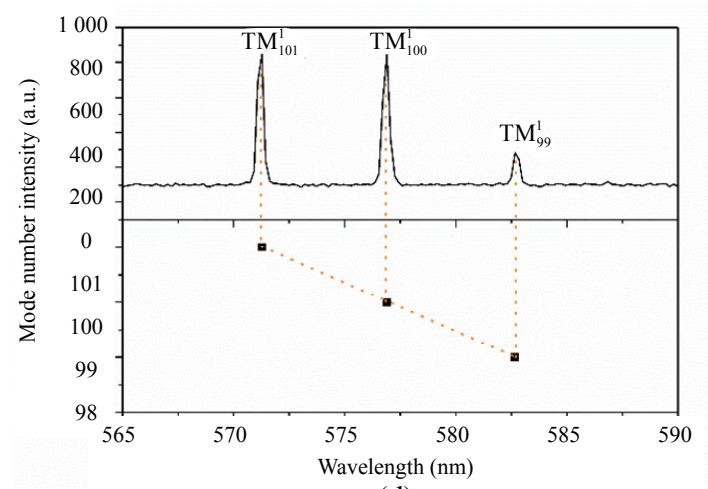

(d)

Fig. 4 Lasing intensity as a function of rotation angle of polarizer on (a) gold substrate and (c) aluminum substrate. Spectra of the fiber under excitation and mode allocation on (b) gold and (d) aluminum. 
In order to obtain more information and study the sensing characteristics, we measured the temperature-dependent spectra of single polymer fiber on two kinds of substrates. Figure 5 depicts the lasing spectra change with various temperatures at pump energy of $5 \mu \mathrm{J}$. Among the two figures, it is clear that laser peaks have a slight blue shift both on gold and aluminum substrates. Both in Figs. 5 (a) and 5 (b), the diameter of single fiber is $\sim 25 \mu \mathrm{m}$. From Fig. 5(a), the temperature sensitivity for polymer fiber on gold substrate is $0.07 \mathrm{~nm} /{ }^{\circ} \mathrm{C}$ when the temperature increases from $26{ }^{\circ} \mathrm{C}$ to $32{ }^{\circ} \mathrm{C}$. With the same situation, the temperature sensitivity for polymer fiber on aluminum substrate is $0.13 \mathrm{~nm} /{ }^{\circ} \mathrm{C}$ when the temperature increases by $6{ }^{\circ} \mathrm{C}$. The wavelength shift is due to the change of both the cavity circumference and the effective index $[5,18$, 19]. The specific change can be described as $[5,20$, 21]:

$$
\Delta \lambda=\lambda_{0}\left(\frac{1}{n} \frac{\mathrm{d} n}{\mathrm{~d} T} \Delta T+\frac{1}{D} \frac{\mathrm{d} D}{\mathrm{~d} T} \Delta T\right)=\lambda_{0} \Delta T\left(\frac{1}{n} \alpha+\beta\right)
$$

where $n, D, \mathrm{~d} n$, and $\mathrm{d} D$ are the refractive index, diameter of the fiber, temperature-induced change in the refractive index, and diameter of the fiber, respectively; $\lambda_{0}$ is the resonant wavelength; $\alpha$ and $\beta$ are the thermo-optic and thermo-expansion coefficients; $\Delta T$ and $\mathrm{d} T$ are the changes of temperature. PMMA has a negative thermo-optic coefficient and a positive thermo-expansion coefficient [5, 22-24]. Thus, the rise in the temperature will cause a decrease in the effective refractive index of the polymer optic fiber, but an increase in the cavity size. The enlargement of the microcavity size promotes redshift of the spectrum [5] while the refractive index decreases which results in the blue shift of the lasing peaks [7]. The final result depends on the competition of them. For the PMMA polymer, the magnitude of the thermo-optic coefficient is much higher than that of the thermo-expansion coefficient [5]. Simultaneously, from Fig. 5, according to the blue shift of the spectrum, the thermally induced negative refractive index change is dominant over the positive thermal expansion [20], and the wavelength shift due to expansion can be ignored.

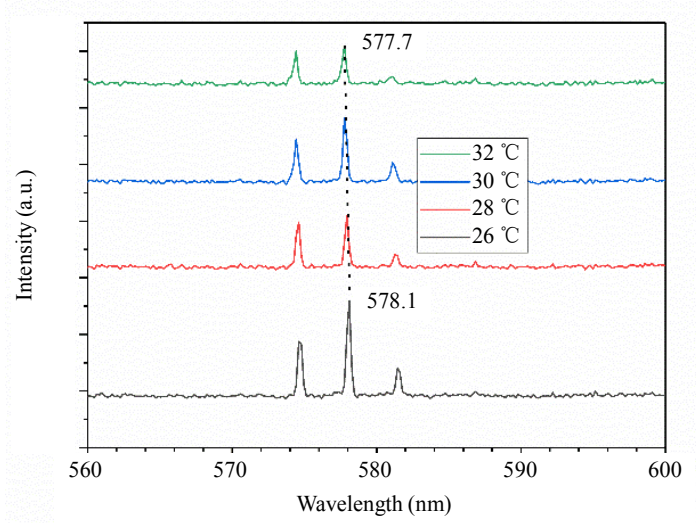

(a)

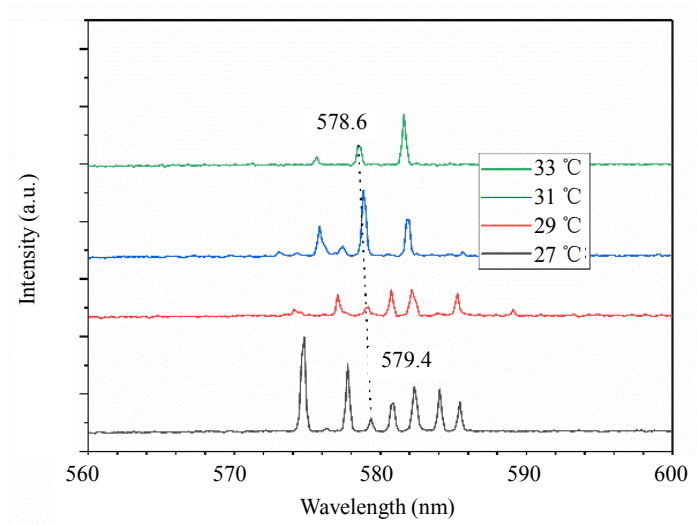

(b)

Fig. 5 Emission spectra of single polymer fiber with $25 \mu \mathrm{m}$ diameter on (a) gold and (b) aluminum substrates at different temperatures.

The quality $(Q)$ factors of polymer fiber WGM microcavities on gold and aluminum substrates are calculated with 1919 and 1 448, respectively. Due to the great metallic absorption, the $Q$ factors of polymer fiber microcavities are very low [25]. The temperature resolution of WGM sensor is proportional to $Q$ factor $[9,26]$, and it can be formulated as $[27,28]$

$$
\Delta \lambda_{\min } / \Delta T_{\min }=(\mathrm{d} \lambda / \mathrm{d} T)
$$

where $\Delta \lambda_{\min }$ is the wavelength resolution of the instrument, $\Delta T_{\min }$ is the temperature resolution of the WGM sensor, and $\mathrm{d} \lambda / \mathrm{d} T$ is the temperature 
sensitivity of the microsphere. In our experiment, $\Delta T_{\min }$ of polymer fiber on gold is bigger than that on the aluminum substrate because of the $Q$ factor. According to the expression above, it is reasonable that the temperature sensitivity of fiber on gold substrate is smaller. So the wavelength shifts of samples on gold substrate are also smaller.

Besides, we studied temperature sensitivity for polymer fiber with different diameters and found that it does not vary linearly with diameter. Figures 6(a)

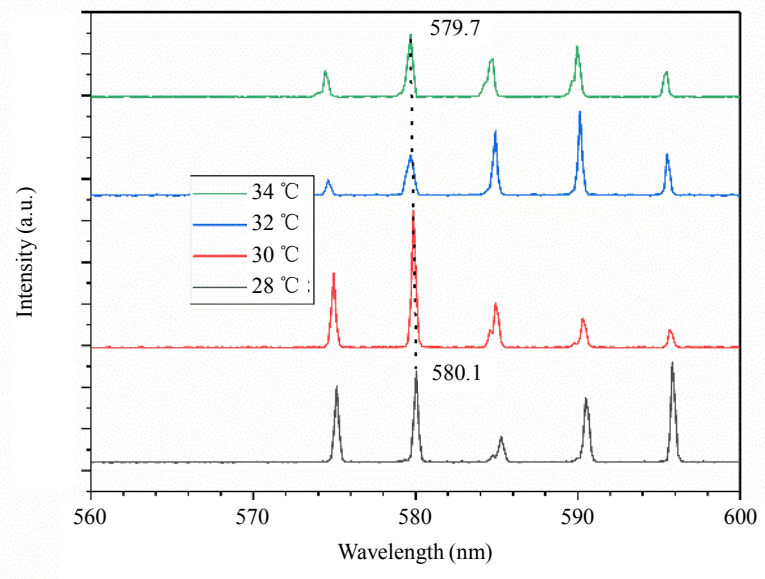

(a)

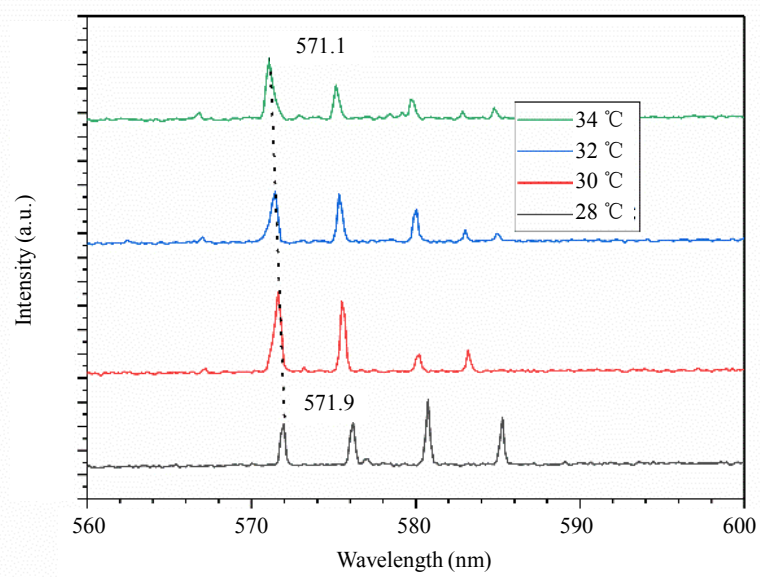

(c) and 6(b) show the WGM lasing spectra of single fiber on gold substrate with diameters of $18 \mu \mathrm{m}$ and $31 \mu \mathrm{m}$. It is clear that the temperature sensitivities are $0.07 \mathrm{~nm} /{ }^{\circ} \mathrm{C}$ and $0.07 \mathrm{~nm} /{ }^{\circ} \mathrm{C}$, respectively. Similarly, the WGM lasing spectra of single fiber on aluminum substrate of $17 \mu \mathrm{m}$ and $35 \mu \mathrm{m}$ are illustrated in Figs. 6(c) and 6(d) with the sensitivities of $0.13 \mathrm{~nm} /{ }^{\circ} \mathrm{C}$ and $0.13 \mathrm{~nm} /{ }^{\circ} \mathrm{C}$, respectively. Therefore, the temperature sensitivity is relatively stable, basically independent of single fiber diameter.

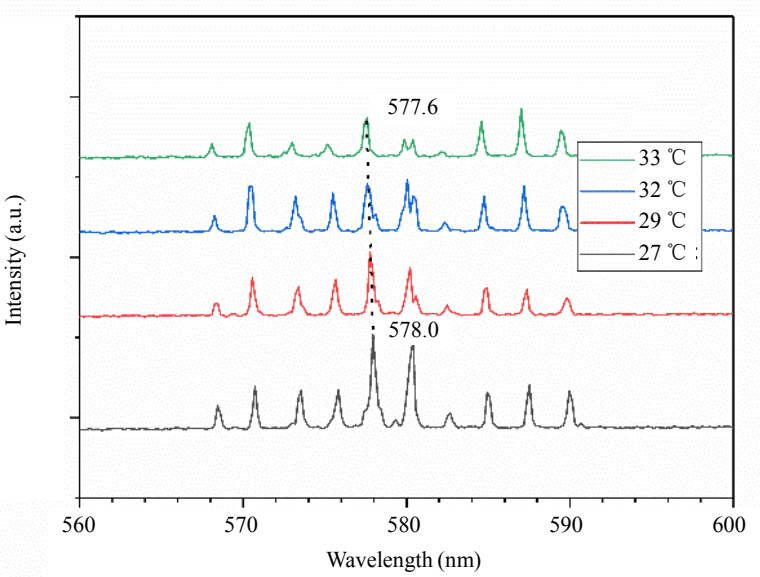

(b)

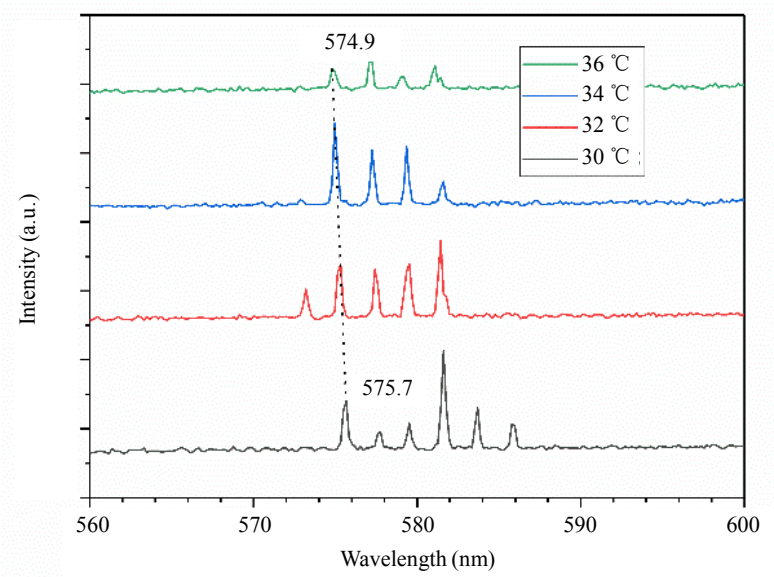

(d)

Fig. 6 Emission spectra of different-diameter single polymer fibers at different temperatures. Samples on (a) and (b) are gold substrates with the fiber diameters of $18 \mu \mathrm{m}$ and $31 \mu \mathrm{m}$ and (c) and (d) are aluminum substrate with the fiber diameters of $17 \mu \mathrm{m}$ and $35 \mu \mathrm{m}$.

\section{Conclusions}

In summary, we fabricated polymer fiber microcavities with the diameters ranging from $4 \mu \mathrm{m}$ to $40 \mu \mathrm{m}$ by electrospinning technology on two different substrates, e.g., gold substrate and aluminum substrate. It has been observed and compared with the SP effect of metal substrate on three aspects of the lasing threshold, lasing emission, and temperature sensitivity of WGM lasers. 
Particularly, it has been found that the thresholds of fibers both on gold and aluminum substrates are smaller than those of most of fiber microcavities, with the values of $0.2 \mu \mathrm{J}$ and $0.4 \mu \mathrm{J}$ on gold and aluminum substrates, respectively. In addition, the polarization characteristics of the WGM lasing are systematically investigated and confirmed, which is in good agreement with the theoretical calculation. Moreover, the temperature sensitivities of polymer fiber on gold and aluminum substrates have been investigated, and the aluminum performs better with the value of $0.13 \mathrm{~nm} /{ }^{\circ} \mathrm{C}$. The metal substrate provides strong optical confinement for WGM polymer fiber microlasers, but brings large absorption losses [25]. In our experiments, WGM polymer fiber microlasers on aluminum substrate have a lower threshold than many results reported in previous studies. And the temperature sensitivity is similarly higher than them. Therefore, it is reasonable that WGM polymer fiber microlasers on aluminum substrate will have a unique application prospect in the field of photonic devices because of its low threshold and good temperature sensitivity. And the electrospinning technique using a simple and particularly low-cost way to fabricate microcavity will be widely used.

\section{Acknowledgment}

The authors would like to thank the financial supports from the National Natural Science Foundation of China (Grant Nos. 12174002, 11874012, 11404087, 11874126, and 51771186); Innovation Project for the Returned Overseas Scholars of Anhui Province (Grant No. 2021LCX011); Key Research and Development Plan of Anhui Province (Grant No. 202104a05020059); The University Synergy Innovation Program of Anhui Province (Grant No. GXXT-2020-052); Project of State Key Laboratory of Environment-Friendly Energy Materials, Southwest University of Science and Technology (Grant No. 19FKSY0111).
Open Access This article is distributed under the terms of the Creative Commons Attribution 4.0 International License (http://creativecommons.org/licenses/by/4.0/), which permits unrestricted use, distribution, and reproduction in any medium, provided you give appropriate credit to the original author(s) and the source, provide a link to the Creative Commons license, and indicate if changes were made.

\section{References}

[1] Z. Zhou, F. Shu, Z. Shen, C. Dong, and G. Guo, "High- $Q$ whispering gallery modes in a polymer microresonator with broad strain tuning," Science China Physics, Mechanics \& Astronomy, 2015, 58(11): 1-5.

[2] R. S. Moshrefzadeh, M. D. Radcliffe, T. C. Lee, and S. K. Mohapatra, "Temperature dependence of index of refraction of polymeric waveguides," Journal of Lightwave Technology, 1992, 10(4): 420-425.

[3] C. L. Linslal, S. Mathew, P. Radhakrishnan, V. P. Nampoori, C. P. Girijavallabhan, and M. Kailasnath, "Laser emission from the whispering gallery modes of a graded index fiber," Optics Letters, 2013, 38(17): 3261-3263.

[4] V. D. Ta, R. Chen, L. Ma, Y. J. Ying, and H. D. Sun, "Whispering gallery mode microlasers and refractive index sensing based on single polymer fiber," Laser \& Photonics Reviews, 2013, 7(1): 133-139.

[5] V. R. Anand, S. Mathew, B. Samuel, P. Radhakrishnan, and M. Kailasnath, "Thermo-optic tuning of whispering gallery mode lasing from a dye-doped hollow polymer optical fiber," Optics Letters, 2017, 42(15): 2926-2929.

[6] C. L. Linslal, M. Kailasnath, S. Mathew, T. K. Nideep, P. Radhakrishnan, V. P. Nampoori, et al., "Tuning whispering gallery lasing modes from polymer fibers under tensile strain," Optics Letters, 2016, 41(3): 551-554.

[7] R. Chen, V. D. Ta, and H. D. Sun, "Bending-induced bidirectional tuning of whispering gallery mode lasing from flexible polymer fibers," ACS Photonics, 2014, 1(1): 11-16.

[8] L. L. Martin, C. Perez-Rodriguez, P. Haro-Gonzalez, and I. R. Martin, "Whispering gallery modes in a glass microsphere as a function of temperature," Optics Express, 2011, 19(25): 25792-25798.

[9] B. B. Li, Q. Y. Wang, Y. F. Xiao, X. F. Jiang, Y. Li, L. Xiao, et al., "On chip, high-sensitivity thermal sensor based on high- $Q$ polydimethylsiloxanecoated microresonator," Applied Physics Letters, 2010, 96(25): 251109.

[10] Y. N. Zhang, N. Zhu, T. Zhou, Y. Zheng, and P. P. Shum, "Research on fabrication and sensing properties of fiber-coupled whispering gallery mode microsphere resonator," IEEE Sensors Journal, 2020, 
20(2): 833-841

[11] C. Xu, F. Qin, Q. Zhu, J. Lu, Y. Wang, J. Li, et al., "Plasmon-enhanced $\mathrm{ZnO}$ whispering-gallery mode lasing," Nano Research, 2018, 11(6): 3050-3064.

[12] S. I. Shopova, C. W. Blackledge, and A. T. Rosenberger, "Enhanced evanescent coupling to whispering-gallery modes due to gold nanorods grown on the microresonator surface," Applied Physics B, 2008, 93(1): 183-187.

[13] P. R. West, S. Ishii, G. V. Naik, N. K. Emani, V. M. Shalaev, and A. Boltasseva, "Searching for better plasmonic materials," Laser \& Photonics Reviews, 2010, 4(6): 795-808.

[14] S. Yang, Y. Wang, and H. Sun, "Advances and prospects for whispering gallery mode microcavities," Advanced Optical Materials, 2015, 3(9): 1136-1162.

[15] C. C. Lam, P. T. Leung, and K. Young, "Explicit asymptotic formulas for the positions, widths, and strengths of resonances in Mie scattering," Journal of the Optical Society of America B (Optical Physics), 1992, 9(9): 1585-1592.

[16] S. K. Tang, R. Derda, Q. Quan, M. Loncar, and G. M. Whitesides, "Continuously tunable microdropletlaser in a microfluidic channel," Optics Express, 2011, 19(3): 2204-2215.

[17] Y. X. Zhang, X. Y. Pu, L. Feng, D. Y. Han, and Y. T. Ren, "Polarization characteristics of WhisperingGallery-mode fiber lasers based on evanescentwave-coupled gain," Optics Express, 2013, 21(10): 12617-12628.

[18] S. Yang, T. Y. K. Eugene, Y. Wang, X. Zhao, H. V. Demir, and H. Sun, "Wavelength tuning of the spirally drawn whispering gallery mode microfiber lasers and the perspectives for sensing applications," Optics Express, 2017, 25(3): 2618-2626.

[19] R. Chen, V. D. Ta, and H. D. Sun, "Single mode lasing from hybrid hemispherical microresonators," Scientific Reports, 2012, 2(1): 1-6.

[20] L. Shi, T. Zhu, D. Huang, and M. Liu, "Thermooptic tuning of integrated polymethyl methacrylate sphere whispering gallery mode resonator," IEEE Photonics Journal, 2016, 8(5): 1-7.

[21] Y. Wang, H. Li, L. Zhao, Y. Liu, S. Liu, and J. Yang, "Tunable whispering gallery modes lasing in dye-doped cholesteric liquid crystal microdroplets," Applied Physics Letters, 2016, 109(23): 231906.

[22] J. M. Cariou, J. Dugas, L. Martin, and P. Michel, "Refractive-index variations with temperature of PMMA and polycarbonate," Applied Optics, 1986, 25(3): 334-336.

[23] C. S. Moreira, A. M. N. Lima, H. Neff, and C. Thirstrup, "Temperature-dependent sensitivity of surface plasmon resonance sensors at the gold-water interface," Sensors and Actuators B: Chemical, 2008, 134(2): 854-862.

[24] Z. Zhang, P. Zhao, P. Lin, and F. Sun, "Thermo-optic coefficients of polymers for optical waveguide applications," Polymer, 2006, 47(14): 4893-4896.

[25] Q. Yao, Y. Huang, Y. Yang, and J. Xiao, “Analysis of mode characteristics for microcircular resonators confined by different metallic materials," Journal of Semiconductors, 2016, 37(12): 124004.

[26] Y. Wu, Y. J. Rao, Y. H. Chen, and Y. Gong, "Miniature fiber-optic temperature sensors based on silica/polymer microfiber knot resonators," Optics Express, 2009, 17(20): 18142-18147.

[27] Q. Ma, T. Rossmann, and Z. Guo, "Temperature sensitivity of silica micro-resonators," Journal of Physics D: Applied Physics, 2008, 41(24): 245111.

[28] Q. Ma, T. Rossmann, and Z. Guo, "Whisperinggallery mode silica microsensors for cryogenic to room temperature measurement," Measurement Science and Technology, 2010, 21(2): 025310. 